$\begin{array}{ll}\text { Research Square } & \begin{array}{l}\text { Preprints are preliminary reports that have not undergone peer review. } \\ \text { They should not be considered conclusive, used to inform clinical practice, } \\ \text { or referenced by the media as validated information. }\end{array}\end{array}$

\title{
Slum Dwellers' Adherence to COVID-19 Guidelines: Application of the COM-B Model to Barriers and Facilitators of Behavioural Change in Uganda's Urban Slums
}

\author{
Gloria Seruwagi ( $\square$ gseruwagi@musph.ac.ug ) \\ Makerere University \\ Hilda Namakula Masaba \\ Centre for Health and Social Economic Improvement (CHASE-i) \\ Agnes Kyamulabi \\ Makerere University \\ Catherine Nakidde Nzesei \\ Centre for Health and Social Economic Improvement (CHASE-i) \\ Daniel Magumba \\ Centre for Health and Social Economic Improvement (CHASE-i) \\ Maria Ssematiko \\ Centre for Health and Social Economic Improvement (CHASE-i) \\ Francis Abura \\ Centre for Health and Social Economic Improvement (CHASE-i) \\ Brian Luswata \\ Ministry of Health \\ Eric Lugada \\ Centre for Health and Social Economic Improvement (CHASE-i)
}

\section{Research Article}

Keywords: COVID-19, adherence, SOPs, slum health, COMB, behavioural change

Posted Date: February 9th, 2022

DOI: https://doi.org/10.21203/rs.3.rs-1295790/v1

License: (9) This work is licensed under a Creative Commons Attribution 4.0 International License. Read Full License 


\section{Abstract}

Following the WHO's global pandemic declaration and Uganda's confirmation of its first COVID-19 case in March 2020, a set of guidelines and directives were given to prevent the spread and mitigate the effects of the novel coronavirus. While initial strict enforcement was ensured and adherence to these preventive guidelines registered, it rapidly declined within a few weeks. This paper uses the ecological systems theory and COM-B model to explore adherence barriers and facilitators. We used data from a mixed methods cross-sectional study conducted in two slums within Kampala, Uganda's capital city. Study participants were 807 ( $660=$ quantitative, 147=qualitative). For data collection we used interviews, focus group discussions, observation and a community survey. Quantitative data was collected using ODK, cleaned, coded in excel and transferred to SPSS for analysis. Qualitative interviews were audio recorded, transcribed, coded and thematically analysed using NVivo12 software. Study findings show high and varied levels of non-adherence with hand washing at $52.7 \%$ and disinfection of touch surfaces at $9.9 \%$. Non-adherence was attributed to a number of economic, sociocultural, political and structural reasons. Despite availability of prevention opportunities, weak capacity and low motivation led to largely undesirable behavior which increased the risk to COVID-19 infections among slumdwellers. The COM-B model can be used to better understand critical intervention pathways to increase adherence to policy and adoption of health protection behavior among vulnerable communities. It is possible to institute adherence barriers among slum dwellers through simple policy and programming responses if contextual factors are recognised.

\section{Background}

\section{COVID-19 in Uganda}

At its onset, the COVID-19 pandemic led to widespread promotion of several preventive and mitigation measures within and across borders $[1,2]$. These included social distancing, handwashing with soap, face masking, sanitizing, avoiding touching of the eyes, nose and mouth, keeping a distance of at least one metre while in public among others [2]. The Uganda national response included all these guidelines in addition to more stringent measures like travel ban and cessation of public gatherings which were enforced from March - July 2020 [3]. The Ministry of Health also instituted a comprehensive behavioural change communication (BCC) campaign in different languages using ICT, print and broadcast media distributed through media across the country [4]. Uganda's national response approach to COVID-19 has been acclaimed from some sections, cited as the reason behind observed lower infection and mortality rates compared to neighbouring countries in the first wave $[5,6,2]$. However, Uganda has also witnessed several and recurring compliance and adherence challenges which have been attributed to several factors. The main reason being that the socioeconomic consequences of the COVID-19 preventive measures for the average Ugandan hit hard; anecdotal evidence shows that many Ugandans defied the regulations in search of daily meals for their families [7]. Additionally, the top-down nature of the health promotion and COVID-19 prevention campaign, coupled with the infodemic and local myths, created mixed feelings regarding the disease and its preventive measures with some pockets of the population believing they were not at risk because of their [African] race [8, 9]. The fact that the pandemic coincided with Uganda's biggest political season heightened the debate as to whether COVID-19 was a politically motivated ploy to undermine some political players in the elections [10, 11 , 7]. Among vulnerable communities like refugees and urban slum dwellers, adherence to preventive guidelines were largely undermined through physical-structural realities which include living in crowded, impoverished setting and the lack of essential items like soap, water for handwashing, face masks and sanitizers [12]. More than $60 \%$ of Kampala - Uganda's urban population dwells in informal settlements most of which are slums [13] being a capital city, Kampala was the epicentre of not only COVID transmissions from the onset but also the prevention and mitigation effort of the national response [14].

\section{The COM-B Model}

Public health emergencies like the Ebola outbreaks in West Africa and Uganda, or pandemics like SARs and COVID-19, often require a behavioural shift within populations to mitigate them [15]. However, behaviour change is complex and rarely linear [16] which is why frameworks like the COM-B model are useful in enabling us to understand the preconditions, processes and outcomes of BCC campaigns. Michie, Stralen and West [17] developed the COM-B model which illustrates that behaviour change is the result of an interaction between three conditions namely: capability, opportunity and motivation. The model posits that for an individual to engage in any behaviour; they need to have the physical and psychological ability (capability), a social and physical context that prompts the behaviour (opportunity), and they should want or need to engage in the behaviour more than any rival behaviours at that moment (motivation). The COM-B model is illustrated in Figure 1 below:

The model has been applied across a range of studies including those developing clinical interventions to improve heart disease diagnosis [18] and the use of hearing aids [19]. Several researchers have also applied it to identify factors that hinder and/or facilitate uptake of preventive behaviours like diets, chlamydia testing for young people, self-care in heart failure patients and healthy lifestyles after gestational diabetes diagnosis among postnatal mothers [20, 21, 22, 23]. Additionally, it has been applied to understand acceptance, 
compliance and adherence to the mostly behavioural preventive measures of the current COVID-19 pandemic. For example, Gibson Miller and team $[24,25]$ used the model to understand factors influencing the uptake of hygienic practices in the early stages of the pandemic in the UK; and reported that promoting and maintaining motivation, social support and awareness of COVID-19 transmission were important facilitators for adoption of hygienic practices. Anderson and others [26] studied pregnant women in the UK and their adherence to social distancing measures using the COM-B model; the authors reported that the model was useful in identifying the determinants for compliance to social distancing behaviour. This paper applied the COM-B model in understanding the factors that hinder or facilitate compliance and adherence to COVID-19 guidelines among urban slum populations in Uganda.

\section{Methods}

The data used in this paper was extracted from a study on the "Adherence, Lived Experiences and Resilient Transformation among "slumdwellers" (ALERTs) in COVID-19. The ALERTs study was conducted between May - December 2020 in the two urban slum settings of Ki-Mombasa and Kabalagala-Kataba within Kampala, Uganda's capital city. It was a mixed-method cross-sectional assessment that utilized qualitative and quantitative approaches to: 1) assess knowledge and attitude; 2) explore local perception of risk; describe implementation of preventive guidelines while determining feasibility, adherence or compliance; 3) identify existing systems in the COVID19 response and determine their efficacy. We used a two-staged cluster sampling procedure with probability proportional to size (PPS) to select 330 participants from each slum for the quantitative arm of the study. Qualitatively we purposively selected 147 participants. The overall sample size was 807 ( $660=$ quantitative, 147=qualitative). Data was collected through interviews, focus group discussions, observation and a survey. Quantitative data was collected using ODK, cleaned, coded in excel and transferred to SPSS for analysis. Qualitative interviews were audio recorded, transcribed, coded and thematically analysed using NVivo12 software. This paper presents mostly data from the study's qualitative arm but also uses quantitative data for triangulational augmentation.

\section{Application of the COM-B model to Policy Adherence}

Barriers and facilitators were identified at all five levels of Bronfenbrenner's ecological systems model [27, 28, 29]. Bronfenbrenner's theory positions individual actions and development into five nested levels of external influence which are the microsystem, mesosystem, the exosystem, the macrosystem, and the chronosystem. It highlights the complexity of interactions across these different systems and their effect on desired outcomes. In line with this, we assessed adherence to COVID-19 preventive guidelines among urban slumdwellers in Kampala at all levels starting from individual level, household, community, district, regional to the national level. We considered the full spectrum of guidelines ( locally known as "The Presidential Directives") and required actions from the public. We identified the diverse actors and enforcement of interventions by different stakeholders at the community, institutional facilities (e.g. health facilities], service and [national] policy level. Finally, we mapped data pertaining to these various levels, actors and actions to the COM-B model's facets of capacity, opportunity and motivation to determine the extent to which the desired behavioural change (i.e. adherence to COVID-19 prevention guidelines) was achieved. Results are presented in line with how the plethora of interventions in the Uganda national COVID-19 response were adhered to by study participants in Kampala's urban slums. Broadly, interventions under each facet have been listed as follows $[30,17,21]$ :

- Capability: Education, training, information sharing, sensitisation, enablement

- Motivation: Persuasion, Enablement \& Modelling - positive behaviour

- Opportunity: Restriction, Coercion \& Punishment, Enablement

- Environmental restructuring - changing the physical or social environment

Overall, we employed a systems approach which involves integrating all aspects of the planning, delivery and use of services into an integrated model that is based on understanding the intentions and actions of people on both the planning-enforcement and publicadherence sides. This is largely because we recognise the need to combine a holistic (macro- and meso-level) systems perspective with a deeply contextualized (micro-level) understanding of processes and people 'on the ground'.

\section{Results / Study Findings}

A key objective of the ALERTs study was to assess the knowledge, attitude and practice (KAP) in slum communities. Overall, we found that knowledge of the symptoms, transmission and prevention of COVID-19 was generally high. However, this had not translated into the required behavioural change. Infact there were high and varied levels of non-adherence to the different guidelines as illustrated in Table 1 below:

Table 1: Self-reported SOP adherence levels among urban slum participants 


\begin{tabular}{|c|c|c|}
\hline $\begin{array}{l}\text { Preventive measure / } \\
\text { SOP type }\end{array}$ & $\begin{array}{l}\text { Adherence } \\
\text { level } \\
\text { (consistent, } \\
\text { self-reported) }\end{array}$ & $\begin{array}{l}\text { Other information } \\
\text { (\% approximate) }\end{array}$ \\
\hline Handwashing & $52.7 \%$ & $\begin{array}{l}\text { Handwashing was a fairly common practice compared to the other preventive } \\
\text { measures, although it was not always with soap }\end{array}$ \\
\hline Handwashing with soap & $46.8 \%$ & The rest did not use soap when washing hands key reason being un-affordability \\
\hline Avoid handshaking & $36 \%$ & $64 \%$ shook hands at least a few times \\
\hline Maintaining social distance & $33 \%$ & $70 \%$ maintained social distance in public only a few times or sometimes \\
\hline $\begin{array}{l}\text { Avoid crowded congregation } \\
\text { areas (outside home) }\end{array}$ & $32.3 \%$ & Majority $(77 \%)$ had travelled using public means at least a few times \\
\hline Wearing face masks in public & $32 \%$ & $35.45 \%$ used masks only sometimes \\
\hline Sanitizing & $30 \%$ & Occasional sanitized, not regular. $31.5 \%$ had never used a sanitizer \\
\hline Disinfect touched surfaces & $9.9 \%$ & $\begin{array}{l}\text { Up to } 35 \% \text { reported to touching different surfaces which could have been } \\
\text { contaminated on a regular basis }\end{array}$ \\
\hline
\end{tabular}

As shown in Table 1, quantitative data shows varying levels of adherence across different types of COVID-19 preventive measures. Findings from the qualitative arm of this study indicated that the drivers of low adherence and non-compliant behaviour included high unemployment rate (over $31 \%$ ), prevailing myths and perceptions, enforcement fatigue, contradictory messaging from leaders across different government structures and sociocultural norms among others. They are explained further across the different facets of the COM-B model in the next section.

\section{Mapping data onto the COMB model}

\section{Barriers: Individual, community, system/facility and national/policy level Physical Capability}

Kampala slums are densely populated and crowded settings. The average household size is between 5-10 people, who typically share very tiny spaces like one or two roomed houses. This makes preventive measures like social distancing extremely difficult to abide by. The excerpts below illustrate this further:

It can't be possible in a home. There are some people that sleep 3 on a mattress. Do you think social distance is possible in such a setting? We are so congested and we can't observe social distance in this area (Female participant, Kataba)

Social distance cannot work because of the nature of our community. We share taps, latrines [toilets], shops, and we survive from our neighbours so we share even the smallest things like match boxes and soap. So social distance cannot work for this congestion in Kataba (Mixed community FGD, Kataba)

The community's living arrangements were confirmed by the leaders:

Social distancing is very difficult because you will find someone in one roomed home when they are 7 or 8 in that one-roomed house (Government leader, Makindye Division)

We have two categories like those with families and then the young single people. You will find several renting many of them. But also, we have people like those from the North or Eastern part of Uganda who can stay like ten people in a single room. This is their way of life (Community leader, Kataba)

The community leader's excerpt above provides insight into some of the reasons for the slum congestion, including affordability among a particular population demography [young or single] and sociocultural norms. The majority of study participants confirmed COVID-related unemployment and lack of incomes as a primary barrier and root cause for the population's non-adherence to preventive measures: 
The reason we find that many people here are congested is because so many are unemployed. You can find someone just goes to a friend's shop and sits there all day long just like the ones that used to work in the bars. If they were all given back their jobs they would care more knowing it's their business but now since it's not their job they don't care much about observing the guidelines like social distance (Youth Girls FGD, Kataba).

Non-adherence to the preventing measure regarding wearing facemasks was due to a number of reasons including the masks not being available or of good quality, being too small to comfortably wear, the belief that they do not protect from COVID-19, low perception of risk driven by lack of exposure to actual cases, the belief that COVID-19 was just a hoax and the perceived availability of alternative protective options:

Like me, I have a problem of allergy, I cannot breathe so well when I put on a mask but because it's an "order from above" I am forced to put on. But I can't do it all day, then there are people who are asthmatic. That's why some other people don't put on masks because they are tiresome. So, they prefer putting Uganda Waragi [local alcohol] in their hands and face to remain safe. They say if a person coughs and sneezes, the alcohol on the face and hands won't let it survive (Male, Parents FGD in Ki-Mombasa).

What is not realistic is telling us to put on masks because there are places where you meet without masks and where you have to shake hands because you know each other... masks don't even save anybody from getting the virus... we ourselves would be in better way to protect ourselves. They gave us substandard masks, some are small, they squeeze the ears, the nose, we have like 6 each and even if I brought them for you, you could see that [they are very small and uncomfortable]. The person who can wear that government mask the whole day deserves a reward (FGD with men, Kataba)

Wearing a mask in Kataba is still a challenge because the masks the government gave out were small and uncomfortable... although we have also had politicians spreading propaganda that COVID doesn't exist, and the hoax thinking started with them that" if COVID truly exists why aren't people dying?" Like I told you we [government] gave out the masks but people don't use them. Even if you go through the community you will not find people putting masks on (Community leader, Kataba).

Adherence to movement restrictions was rendered almost impossible by the need to secure livelihoods and food security. In slum communities, most people's jobs are located in crowded settings and require a physical presence, movement and human interaction. Pandemic restrictions heightened the need for food and other basic needs for survival which had significantly reduced in most communities due to lack of income from pandemic-related unemployment and business closures. People had to make some difficult decisions regarding food and livelihoods, inevitably contravening SOPs:

In this corona families are broken down because of poverty. The husband leaves home because of misunderstandings, he simply cannot stay there because there are many quarrels at home. Since there is no income some people have found challenges paying rent to their landlords so they can't stay home, they must move and look for what to eat (VHT, Kataba)

During the lockdown very many people needed food. They had not been working and the interventions of food distribution by the government did not reach every homestead, so .... people rushed back to work and cared more about their survival than the SOPs (Implementing Partner/IP NGO, Ki-Mombasa)

\section{Psychological Capability}

The lack of sufficient and accurate information about the pandemic, especially at its onset, significantly contributed to the community's non-adherence to preventive measures. Nearly all community study participants reported not having witnessed a COVID-19 case and so did not believe it was real. This was partly because of the nature of facility-based COVID-19 management, where suspected or confirmed cases were promptly isolated and/or quarantined outside of the community. As a result, many people held many beliefs including that the pandemic was a political gimmick and a hoax during the political election season, an invented "cash cow" for unscrupulous government officials bent on getting money from potential donors. A lot of information was reported to have been received from the media especially social media which, showed high fatalities in western countries other than Africa. Based on this some study participants believed that the African race was immune to the disease suspected to be a form of depopulation ploy by racial supremacists. Moreover, prevention messages did not seem to have sufficiently educated and informed the masses regarding the pandemic. These myths, falsehoods and local beliefs are illustrated in the excerpts below:

The challenge with us Africans is that we believe by seeing... so when people learn that somebody has died of COVID-19, they then take it seriously. They hear of people dying in other countries but haven't seen anyone dying here in Uganda. They see that and believe that their blood is different from others and we are stronger since we are Africans (Local leader, Kawempe) 
Many of them were refusing to accept it has come to Uganda. They thought it was a political stunt and that the president was the one trying to control people. I tried hard to show especially the elderly ones that if this disease is killing people in our neighbouring countries then it sure wont spare us since we are all human. Unfortunately, people still up to now believe that after elections we won't have this disease anymore (Security Personnel, Ki-Mombasa).

Personally, I have not seen a covid patient with my eyes, only on TV.... we protect ourselves by wearing masks, washing our hands, keeping social distancing as you have been announcing on TV, as a preventive measure, but still we are doing that without knowing what we are preventing. It is like you put on a condom knowing your preventing yourself from HIV, but here we wash hands, but you don't know where it is going to come from. If it is airborne and was targeted for Africa, even if you wash hands and the legs and your partner sleeps at a distance, still you will die. This is an air borne thing. Why don't you wash the noses because they are the ones that inhale? Hands don't inhale, how do you tell me to wash hands when I am using the nose to inhale? This is comedy, because I am using my nose to breathe, Covid 19 is designed to depopulate us (Male participant, Kataba)

As part of Uganda's national response, Ugandans had multiple, and sometimes contradictory, message centers regarding COVID-19 infection. In addition to behavioural communication messages sent through the mainstream media, local communities received messages from law enforcement officers (the police), Village Health Team (VHT) members, community leaders, politician, and locally operating NGOs. This diversity in messaging and messengers was reported to have confused people who did not know what to believe or follow.

They talk about it [the pandemic] but it's hard to believe, because the president says this today and the doctors say another thing. So, we don't know what to believe and what not to believe. The majority have given up on corona and they no longer care. Those who cares will just use local herbs to treat it, after all it is one of the things, they are telling us to do (Parents FGD, Ki-Mombasa)

\section{Reflective Motivation}

Beliefs regarding perceived risk entailed the perception that certain population categories are more at risk than others. For example, children and young people were generally not regarded as high-risk groups, so there was a laxity in targeting them with information to ensure adherence in this group. This was exacerbated by prevailing myths and falsehoods regarding the COVID-19 infections as well as limited exposure to actual cases:

They still think that maybe COVID-19 is a myth, it's a political thing. Then you realize that there are a number of youths in the slum areas and youth think that they're still strong enough so they can fight the disease. They also think that the disease is for the elderly, people who have co-morbidities and all that (Local government official, Makindye Division)

It hasn't been easy because when you are educating them about COVID they ask you "who has died of it?" so they got tired of the prevention message so it hasn't been easy (Village Health Team /VHT member, Kataba)

The period between 2020 and 2021 was a political season in Uganda. Since it coincided with the pandemic the community was highly suspicious of the intentions of the political leaders. Highest among the suspects was the ruling government, it contested for continued leadership which had already span a period of over three decades, it was accused of using COVID-19 as an excuse to decimate the opposition's political campaigns. While corruption is a well-documented vice in Uganda, the public was concerned that designated enforcers of preventive measures, such as the police and other security personnel, were more interested in collecting bribes from those caught contravening SOPs than supporting implementation of the national COVID-19 response. As a result, the motivation to comply with preventive guidelines was extremely low:

We have these laws but they are not followed ... enforcers arrest you then you pay them to release you and those that fail to pay are the ones they detain (Community member, Ki-Mombasa).

So, like $70 \%$ of the people think Covid is not there because it has been politicized by the leaders. The same people in leadership like the president and ministers conduct campaigning rallies with many people and they made people think it was all a lie. In this period of campaigns, you find the politicians coming to do rallies and they gather people, give them incentives without even minding about social distancing and other SOPs (Youth Leader IDI, Ki-Mombasa).

People know about COVID but they also have this propaganda that it is all a lie. Who spreads the propaganda that COVID is a lie to these people? Politics, it's just that they are people who don't like the ruling government so whatever they tell us is a lie to them. So, when politics came in, and they saw that the ruling party doesn't care much about COVID, people started having doubts. Also, when even the Minister of Health Dr. Acing herself went back to her area to campaign, in the middle giving us the SOPs of COVID on national television, she didn't 
show an example to us. That means there are in for their political gains to get money that was being given to countries affected with COVID. So, people begun to take the COVID issue lightly (Community Leader, Kataba)

\section{Automatic Motivation}

Study participants reported the fear and shame to seek help or healthcare for suspected cases as a major barrier. Initially people with related symptoms were advised to report to health facilities for medical attention or call a toll-free line. However, there was a lot of stigma and anxiety surrounding testing, isolation and subsequent quarantine processes which was more like a "black box" with limited public awareness with cases were managed away from the community. This led to fear of ostracization for suspected or confirmed cases the study findings showed that some people did not report or seek care as expected. This implied that that some hitherto preventable transmission was inevitable and enabled by the culture of silence. The fear of receiving a confirmed positive result after testing meant that some cases remained undetected and continued to spread into the community:

We had someone we suspected to have the virus because he had just arrived from Dubai and we were questioned as to why we couldn't call the Ministry of Health yet we had the Tollfree numbers. The people also feared to be taken into isolation as contacts to this person that's why they did not make the call (Youth Girls FGD, Kataba).

\section{Social Opportunity}

Related above, the stigma associated with testing positive for COVID-19 seemed to outweigh the benefits of practicing healthy and preventive behaviour such as testing, seeking medical care, correctly and consistently wearing facemasks or social distancing. Participants did not think that anyone would sympathize with them, they would be perceived to have "broken the rules in the first place" reason they caught the virus. In addition, sociocultural norms acted as barriers to prevention. For example, guidelines like social distancing were a diversion from deeply cherished sociocultural norms, particularly those deemed essential when affected by disasters such as a pandemic. Rituals like greeting, touching, hugging and eating together with a loved one in trouble or in pain are highly regarded as therapeutic and socially connecting. Therefore, a number of people were not entirely comfortable to abandon their cherished practices in a situation where they were even deemed essential.

There are things that can change and there are those that cannot change. Things like hugging and shaking of hands are no longer done much but as people from the East like the Bisaya, Alurs and Acholi we eat together and it's our culture (Community leader, Kataba)

Related to this innate need to socialize and connect was peer pressure and social desirability. Study participants highlighted the need to fit in with peers and other groups deemed important to them, even though some of norms were not compatible with preventive guidelines. For example, many youths were not wearing face masks since they wanted to fit in and not be perceived as deviant, this affected the overall mask wearing practice. In addition, politicians on the campaign trail tried to show their electorate that they were not "scared" of them even when not protected with masks or distance in an attempt to display unity as "one" during vote canvassing. Moreover, many local leaders and enforcers of preventive guidelines were themselves not adhering to the guidelines. The police action of arresting those contravening the regulations and loading them in a crowded prison cells without any protection was also counterproductive and contributed to an increased case load.

Our leaders have done nothing in regards to that [prevention]. They are not leading by example, because even at their homes there are no hand washing facilities which they want us to have. They are the ones also moving around with no masks on, even the police who come to arrest others they be there having no masks. And you see how politicians are going to their campaign rallies without anything on for themselves or their supporters. Sincerely how can you take these people seriously? (FGD Community, Kataba)

Other notable barriers were religious in nature where preventive guidelines were in disharmony with people's religious beliefs or the need to congregate for fellowship. For example, the Muslim community reported that use of alcohol-based sanitizers was favored by their community. Although the religion factor was noted to be double-edged facilitating adherence in some aspects of the pandemic. For example, some communities' religious practices (specifically Islam) were aligned to COVID-19 preventive guidelines like swift burial, covering the face or masking, frequent handwashing and maintaining overall hygiene.

\section{Physical Opportunity}

As already highlighted among the barriers for physical capability, the living arrangements and household setup in slums presented massive impediments for adherence to COVID-19 preventive measures. Overcrowded households and intense social mixing as a way of life made it impractical for physical distancing. There is also a likelihood that this close contact state resulted in high infection rates in a very short time. Furthermore, slum settings generally have inadequate WASH infrastructure, limited resources, risky livelihoods and extremely low access to healthcare: 
Most of the people here rent so they share the toilet facilities and water sources. Unless the landlord is able to build more toilet structures, very many people use a single toilet. And also, we have many prostitutes in here Kataba so they would not really mind social distance when they are looking for customers (VHT, Kataba)

Our place here is a ghetto. In a ghetto, we grow up as one family. You eat together, move together, so it is difficult to tell a friend you have stayed with for over 20 years that you extend and keep distance, yet we have grown up with that solidarity of brotherhood, that oneness. Secondly, looking at the ghetto setting, we stay close to each other, it is one door after the other. Immediately you step out of your room, you bump into a friend and greet them. So, it is so hard to maintain social distances (Youth FGD, Ki-Mombasa)

There are some places where you go and find many people but without a jerrycan of water to wash hands. There are water points where they fetch water. Of course, many of them do not have water connected to their homes. Even if there is a tap, many of them have to go to the same tap. Then some of them share toilets and bathrooms. As for the masks, some even take weeks without wearing them (Local Government Official, Makindye Division)

In summary, the slum communities of Ki-Mombasa and Kabalagala-Kataba reported several barriers that hindered adherence to COVID-19 preventive measures. These barriers are illustrated, across components of the COM-B model, in Figure 2 below:

Despite these seemingly intractable barriers, there were a number of community members who adhered to COVID prevention measures. Reported adherence facilitators are summarised in Figure 3 below alongside the barriers expounded further in Table 2:

Table 2: Barriers and facilitators of adherence to COVID preventive measures in Uganda's urban slums

Note: Some barriers overlap (e.g. health system level factors have both community and policy angles) 


\section{Psychological Capacity}

Individual, household, community level

- Increasing knowledge and awareness through intensified sensitization and behavioural change communication (BCC) campaigns

- Progressive interface with COVID-19 (C-19) cases, and extensive profiling by the media

Policy level

- Clear SOPs and guidelines e.g. on screening, testing, isolating, quarantining, referral and management of patients

- Ongoing information sharing on C-19, SMS message prompts

\section{Physical Opportunity}

Individual, household, community level

- Mass distribution of free masks by government and partners

- Relief and food distribution to some vulnerable households

- Intensified WASH campaigns, more infrastructure and locallydriven equipment

Policy level

- The way the national C-19 response was organised (equipping higher level health facilities to manage cases)

\section{Social Opportunity}

Individual, household, community level

- Social norms and culture (positive) e.g. dresscode for women, handwashing and swift burial among Muslims

- Some influencers led by example e.g. local celebrities and leaders

\section{Policy level}

- Targeted BCC campaigns and periodic updates

- Quick resource mobilisation from various stakeholders in all forms
Individual, household, community level

- Community perceptions, assumptions, myths and falsehoods

- Limited provider knowledge about the epidemiology and management of C-19, and treatment options (at onset)

Policy level

- Limited practical guidance and information sharing, especially for public lower level health facilities (LLHFs)

- Lack of guidance and/or trust in the intentions and actions of government

- Framing of BCC messages was not designed with slum communities in mind - e.g. social distancing was impractical

Individual, household, community level

- Ill-fitting masks and related discomfort

- Existing health conditions e.g. asthma and other breathing complications

- Limited WASH infrastructure and tools e.g. soap and water

Policy level

- Shortages in health workforce
Individual, household, community level

- Social norms and culture (harmful in light of C-19)

- Limited supplies (PPEs), space and certain services at health facilities

Policy level

- Institutional / organisational culture and longstanding practice norms, encouraged by policy e.g. referral vs. case management at health facilities

- Case management required separation of loved ones which people loathed and it limited utilisation of healthcare services

\section{Reflective Motivation}

Individual, household, community level

- Increasing risk perception with rising cases

- Belief that adherence to "the more-feasible" guidelines is protective

- High confidence - from skills-based training and higher-level cadre, raised self-esteem and empowerment as well as confidence to support community-based surveillance teams

Policy level

- Targeted messaging along the way e.g. for children and youth
Individual, household, community level

- Limited exposure to C-19 cases (at onset)

- Myths, falsehoods, social media, infodemic

Policy level

- Policy approach to health system preparedness: Focused and phased approach to response meant that LLHFs and HCW had no knowledge, basic supplies, capacity, experience or resilience

- Feasibility: Some guidelines not feasible and perceived to be unrealistic in crowded slum settings 


\section{Facilitators}

Individual, household, community level

- Increasing community transmissions and registered deaths

- Limited health system capacity and complex referral process

- Policy push for home-based care model

Policy level

- Rewards and incentives - protection for self and others; the inconvenience of punishment for being caught in contravention (jail, bribes, beatings)

- Handouts - mask distribution, food distribution

- Feedback and periodic information sharing with service providers across the health system level motivated them to continue enforcing adherence in their areas of jurisdiction

\section{Physical Capability}

Individual, household, community level

- Training: Some HCWs had acquired skills-based training, including simulation and were prepared to handle any C-19 case or emergency

Policy level

- History of managing epidemics and existing infrastructure in some health facilities.

- Study sites relative proximity to equipped high level health facilities, including national referral hospitals

- HF level in light of Uganda's health system design; especially referrals

\section{Barriers}

Individual, household, community level

- Fear of infection and perception of risk - that community would infect them (police helpful in enforcing) and that HCWs are at risk

Policy level

- Testing and isolation/quarantine approach - testing results take long creating more anxiety; and procedure once confirmed positive made people feel uncomfortable

- Limited availability and proximity, high cost for testing
Individual, household, community level

- Lack of appropriate and sufficient training and skills

- Limited supervision and support from for LLHFs

- Shortage of PPEs and other critical equipment/supplies, health care workers were not operating optimally

- Infrastructure and general health facility preparedness - spacing ventilation, staffing for different (triage, isolation)

Policy level

- Health system design and preparedness (across the blocks)

- The mode of enforcement / enforcer interpretation and implementation approach - mostly top down and involving uncoordinated multiple power centres

- Context - the political season and leaders not leading by example

- Testing - uncomfortable, costly

\section{Psychological Capability}

Individual, household, community level

- Increasing knowledge, awareness and education - as C19

info became more aware

- Goodwill from the HCWs - irrespective of their lamentable working conditions

Policy level

- President's leadership and direct involvement - combination of carrot and stick approach

- National policy and health system design - both a barrier and facilitator; double edged.
Individual, household, community level

- National policy and health system design (tiered with a strong referral component, equipped based on level and complexity of cases)

- Design of the national covid response (decentralised and functional taskforces with multiple stakeholders including nonhealth (political leaders, law enforcement, etc)

Policy level

- National policy and health system design - both a barrier and facilitator; double edged.

\section{Cross-cutting Factors}

- Engagement of community and local leaders

- Sensitisation and addressing stigma, especially by local leaders
- Limited confidence and anxiety on LLFs facilities to effectively manage $\mathrm{C}-19$ cases

\section{Discussion}


Study findings highlight variations in adherence levels across different types of COVID-19 preventive guidelines and SOPs. Associated explanatory factors were also varied but mostly pointed to the feasibility and practicality of some guidelines and SOPs compared to others, within the context of informal settlements and more specifically the urban slums. Despite this study's findings which shows that adherence to handwashing ranks comparatively higher than other guidelines, it is still lower than the protective threshold for COVID-19 infection; evidenced from other studies [31,32] which showed urban slums to have had significant WASH and other challenges. Contextual appropriateness, whether perceived or actual, is critical to the success of any behavioural intervention [33,34,35]. Previous studies [36, 37] highlighted how Kampala slums required special support to counter the drivers of COVID-19 infection risk associated with living conditions.

There is a notable level of consistency between community knowledge, attitude, practice (KAP) and behaviour in regard to SOP adherence. This knowledge-behaviour link is well documented $[38,39]$ and is integral to this paper's COM-B model. Other determinants, particularly both structural and systemic, are just as powerful barriers or facilitators as the cognitive ones [40,41]. Therefore, addressing both, and indeed all forms of, barriers to adherence should be the goal of all actors seeking to achieve the intended outcomes of an impactful national response to COVID-19.

The paradox of enablers-barriers such as multiple BCC centres, religion, media, diaspora, culture and Uganda's health system design and the national response to COVID-19 approach are equally important adherence influencers. Critically these double-edged factors need to be optimally harnessed for positive behavioral change with their effectiveness closely monitored. Overlap across the different COM-B components and sub-components demonstrate the complexity of human behaviour, its non-linear progression and chaotic pathway [21] must be accounted for while designing policy and programming interventions.

A lot can be learned about formulating, implementing, and evaluating quick and swift policy guidelines when faced with epidemics and pandemics. In the case of COVID-19 there isn't much room for error and flexibility as cases rapidly increase yet with unknown disease outcomes. Contrary to the known routine policy process of "muddling" through with marginal incremental changes [42, 43], the need for precision is even higher in this case where each decision could have lethal implications. Although policy response did achieve significant success, there were gaps and missed opportunities in the context of slum dwellers. Particularly the need to lead by example, transparency and limited community engagement which were amplified by a heavy political campaign season and limited livelihood options. This affected consensus building and rallying of credible coalitions to support securing optimum interventional agreements among various stakeholders. [44, 33].

The responsibility of implementing and monitoring Presidential Directives was given to multiple stakeholders, mostly holding different power centres, reporting lines, training, resources and perceived clout. Whereas the intentions of this concerted multi-level and multipronged effort to enforce adherence to guidelines were good and ideally should have resulted in instant and positive behavioural change, it led to a different and undesirable outcome of suboptimal adherence increasing government mistrust. First, the interpretation of the Presidential Directives was subjective, demonstrated in the diversity of enforcement approaches across the different enforcers who included law enforcement and security personnel, politicians and healthcare teams among many others. Secondly, various actors were not well coordinated or in agreement to the best course of action in case of non-adherence; infact some of the enforcement measures were counterproductive and ill-informed with some enforcers perceived as illegitimate. "Street level bureaucracy" model of implementation $[33,45]$ is complex enough even in the absence of diverse multiple power centres and approaches. The uncoordinated training, perceived illegitimacy and multiple preventive methods adopted by diverse enforcers exacerbated an already fragile health policy framework. There were other factors that potentially competed for action such as breakdown of protective systems, social cohesion, national identity, unificaty in the face of a heated political season, limited availability of mental health services and psychosocial support (MHPSS). It is therefore not surprising that non-adherence was high, partly in defiance to the approaches the various actors adopted evidenced by message framing and source credibility which was directly associated with behavioural intention and action [462,47].

\section{Implications for Policy and Practice}

The policy and health system phased and hierarchical approach to health system strengthening (HSS) was necessary and potentially impactful, however findings from this study indicated that it was a barrier to adherence, partly because the approach was not well understood and applied among urban slum settings. Limited policy knowledge and comprehension among communities of a low socioeconomic status is not uncommon $[48,49]$. The notion of community engagement and involvement (CEI) is however gaining traction as it has been proven over time as an effective strategy for instant, transformation and sustained behavioural change [50, 51]. As the COVID-19 pandemic continues to evolve globally and nationally, it is critical to better understand the CEl, achieve buy-in, ownership and aim to achieve impactful implementation in support of national policy. In addition, a more equitable HSS across all levels of Uganda's healthcare system is required - especially at the grassroot and among lower-level health units to enable a meaningful contribution to effective prevention and case management at all levels [14]. Communities should be held accountable, allowed space to implement with

Page 11/17 
their capacity to monitor and assess implementation progress enhanced. They should be encouraged and challenged to provide localised and sustainable solutions for effective implementation progress of policy initiatives like the national response to COVID-19.

Educational awareness interventions for slum dwellers should focus on feasible and contextually appropriate options rather use of generic prevention message a one-size-fits-all messages which could potentially be interpreted as a mockery of their living conditions. For example, emphasis could be placed on handwashing with soap and wearing masks instead of use of sanitizers and social distancing. The messaging should consolidate the success previously registered from longstanding awareness campaigns that positively evolved to include targeted messaging for different population strata such as children.

While discussed in most policy documents, the essence, and benefits of a truly multisectoral approach remains elusive. Yet COVID-19 has shed light on sectoral interconnectedness and how this can be a critical and impactful intervention influencer. Policy response to public health and cross-sectoral threats like the COVID-19 pandemic require a multisectoral and system approach. They would also require intentional linking and signposting to other actors and institutions with capacity to address potential barriers seemingly out of scope in a specific population segment such as the pursuit of livelihood or food security that potentially impacts a health issue like COVID-19 or teenage pregnancy. All the different sectors involved (such as health, protection, livelihoods or education) need to collaboratively coordinate an appropriate response - first by auditing all available actors, actions and resources before building referral and cross-sectoral signposting systems which are robust.

\section{Conclusion}

Behavioural, environmental, social and system interventions have proven crucial in reducing COVID-19 transmission as well as protecting populations and healthcare systems. Yet adherence to these interventions regarding policy and preventive guidelines continues to prove difficult in urban slums. The COM-B model can be deployed to understand critical intervention pathways for increased policy adherence and health protection for vulnerable communities. Adherence barriers among slums dwellers can be address with simple policy and programming responses if contextual factors are taken into account.

\section{Declarations}

\section{Ethics approval and consent to participate:}

Ethical approval for this study was provided by Makerere University School of Social Sciences Research and Ethics Committee (MASS RES 07.20.420) and the Uganda National Council of Science and Technology (SS555ES). The study also obtained clearance and permission for data collection from the Directorate of Public Health and Environment, Kampala City Council Authority (DPHE/KCCA/1301). Informed consent was obtained from all study participants, and all methods were performed in accordance with the relevant guidelines and regulations in our study, including the 1975 Declaration of Helsinki.

\section{Consent for publication:}

Not applicable.

\section{Funding:}

The ALERTs study grant was funded by the Government of Uganda, through the Makerere University Research and Innovations Fund (MakRIF).

\section{Availability of data and materials:}

All data generated or analysed during this study are included in this published article.

\section{Acknowledgements:}

We are grateful for the contribution of the ALERTs study and field teams. We also thank study participants at the national level, in the KiMombasa and Kabalagala-Kataba sites. Finally, we acknowledge the contribution of our beloved Co-Investigator, Prof. Stephen Lawoko (RIP), who passed away before completion of this manuscript.

\section{Authors' contribution:}


GS, CNN, HNM and EL conceptualised the study. AK, DM, FA, MS and BL participated in data collection, analysis and write up. GS wrote the manuscript's first draft, with all authors providing feedback, refining subsequent versions and approving the final version.

\section{Competing interests:}

The authors have no competing interests to declare.

\section{References}

1. Hillier D, Newton-Lewis T, Nair R, Larsen C. Initial COVID-19 responses in Bangladesh, Kenya, Pakistan, Sierra Leone and Uganda: Documentation and learning from March to May 2020. Maintains, Oxford Policy Management, Oxford, UK. 2020.

2. Federica M, Pattnaik A, Jordanwood T, Nakyanzi A, Byakika S. "Case Study: The Initial CoVID-19 Response in Uganda." Washington, DC: ThinkWell and Ministry of Health Uganda. 2020.

3. Observer Team. Museveni imposes 14-day COVID-19 lockdown. The Observer [Internet]. [cited 01/07/2021]. Available at: https://observer.ug/news/headlines/ 64074-museveni-announces-14-day-covid19-lockdown. 2020.

4. Ajari EE, Kanyike AM, Ojilong D, Abdulbasit IO. COVID-19 in Uganda: Epidemiology and Response. European Journal of Medical and Educational Technologies. 2020 May 14;13(2): em2009.

5. Kitara DL, Ikoona EN. COVID-19 pandemic, Uganda's story. The Pan African Medical Journal. 2020;35(Suppl 2).

6. Sarki AM, Ezeh A, Stranges S. Uganda as a role model for pandemic containment in Africa. American Journal of Public Health. 2020 Dec;110(12):1800-2.

7. Anguyo I, Storer L. In times of COVID-19 Kampala has become 'un-Ugandan'. LSE Blogs. Available at: https://blogs.Ise.ac.uk/africaatlse/2020/04/09/kampala-epidemic-un-ugandan-society-in-times-covid-19/2020.

8. Kaitiritimba R. An effective response to COVID-19 is an inclusive response: the case of Uganda. UHC2030 Blog. Available at: https://www.uhc2030.org/blog-news-events/uhc2030-blog/an-effective-response-to-covid-19-is-an-inclusive-response-the-case-ofuganda-555370/ 2020

9. Kasozi KI, MacLeod E, Ssempijja F, Mahero MW, Matama K, Musoke GH, Bardosh K, Ssebuufu R, Wakoko-Studstil F, Echoru I, Ayikobua ET. Misconceptions on COVID-19 risk among Ugandan men: results from a rapid exploratory survey, April 2020. Frontiers in public health. 2020 Jul 28; 8:416.

10. Schwikowski M. Uganda's unequal political campaigns during COVID-19 times. DW. Available at: https://www.dw.com/en/ugandasunequal-political-campaigns-during-covid-19-times/a-54488099 2020.

11. Tallio V. How the COVID-19 may influence the next Ugandan Presidential elections: an analysis of the impact of the containment measures. ORF. Available at: https://www.orfonline.org/expert-speak/how-the-covid19-may-influence-the-next-ugandan-presidentialelections/ 2020.

12. Lirri E. Dilemmas of Preventing COVID-19 among Kampala's Poor Urban Dwellers. Available at: https://www.kas.de/documents/280229/8800435/COVID19+\%26++Kampala\%E2\%80\%99s+Poor+Urban+Dwellers_Lirri.pdf/5a5538af311a-1543-d918-80cf54c64f9a?version=1.0\&t=1590587203796 2020.

13. Richmond A, Myers I, Namuli H. Urban Informality and Vulnerability: A Case Study in Kampala, Uganda. Urban Science [Internet]. MDPI AG; 2018; 2:22. Available from: http://dx.doi.org/10.3390/urbansci2010022

14. Seruwagi G, Nakidde C, Otieno F, Kayiwa J, Luswata L, Lugada E, Ochen EA, Muhangi D, Okot B, Ddamulira D, Masaba A \& Lawoko S (2021) Healthworker preparedness for COVID-19 management and implementation experiences: a mixed methods study in Uganda's refugee-hosting districts. BMC Confl Health 15, 79 (2021). https://doi.org/10.1186/s13031-021-00415-z

15. Michie S, West R. Sustained behavior change is key to preventing and tackling future pandemics. Nature Medicine. 2021 May;27(5):749-52.

16. Bavel, JJV, Baicker, K, Boggio, PS et al. Using social and behavioural science to support COVID-19 pandemic response. Nat Hum Behav 4, 460-471 (2020). https://doi.org/10.1038/s41562-020-0884-z

17. Michie S, Stralen MM, West R. The behaviour change wheel: A new method for characterising and designing behaviour change interventions. Implementation Sci, vol. 6 (1). doi:10.1186/1748-5908-6-42.

18. Barker F, Atkins L, de Lusignan S. Applying the COM-B behaviour model and behaviour change wheel to develop an intervention to improve hearing-aid use in adult auditory rehabilitation. International journal of audiology. 2016 Jul 8;55(sup3): S90-8.

19. Jatau Al, Peterson GM, Bereznicki L, et al. Applying the Capability, Opportunity, and Motivation Behaviour Model (COM-B) to Guide the Development of Interventions to Improve Early Detection of Atrial Fibrillation. Clinical Medicine Insights. Cardiology. 2019; 
13:1179546819885134. DOI: 10.1177/1179546819885134.

20. Timlin D, McCormack JM, Simpson EE. Using the COM-B model to identify barriers and facilitators towards adoption of a diet associated with cognitive function (MIND diet). Public Health Nutrition. 2021 May;24(7):1657-70.

21. McDonagh LK, Saunders JM, Cassell J, Curtis T, Bastaki H, Hartney T, Rait G. Application of the COM-B model to barriers and facilitators to chlamydia testing in general practice for young people and primary care practitioners: a systematic review. Implementation Science. 2018 Dec;13(1):1-9.

22. Whittal A, Störk S, Riegel B, Herber OR. Applying the COM-B behaviour model to overcome barriers to heart failure self-care: a practical application of a conceptual framework for the development of complex interventions (ACHIEVE study). European Journal of Cardiovascular Nursing. 2021 Mar;20(3):261-7.

23. Boyd J, McMillan B, Easton K, Delaney B, Mitchell C. Utility of the COM-B model in identifying facilitators and barriers to maintaining a healthy postnatal lifestyle following a diagnosis of gestational diabetes: a qualitative study. BMJ open. 2020 Aug 1;10(8): e037318.

24. Gibson Miller J, Hartman TK, Levita L, Martinez AP, Mason L, McBride O, McKay R, Murphy J, Shevlin M, Stocks TV, Bennett KM. Capability, opportunity, and motivation to enact hygienic practices in the early stages of the COVID-19 outbreak in the United Kingdom. British journal of health psychology. 2020 Nov;25(4):856-64.

25. Gibson-Miller J, Zavlis O, Hartman T, McBride O, Bennett K, Butter S, Levita L, Mason L, Martinez AP, McKay R, Murphy J. Psychological Factors Influencing Protective Behaviours during the COVID-19 Pandemic: Capability, Opportunity and Motivation.

26. Anderson E, Brigden A, Davies A, Shepherd E, Ingram J. Capability, Opportunity and Motivation of pregnant women to enact social distancing behaviour in the Covid-19 pandemic in the UK: A qualitative interview study.

27. Bronfenbrenner U. Toward an experimental ecology of human development. American psychologist, 1977. $32(7), 513$.

28. Bronfenbrenner U, Evans GW. Developmental science in the 21st century: Emerging questions, theoretical models, research designs and empirical findings. Social development. 2000 Feb;9(1):115-25.

29. Guy-Evans O. Bronfenbrenner's ecological systems theory. Simply Psychology. www.simplypsychology.org/Bronfenbrenner.html. Accessed $5^{\text {th }}$ November 2021

30. Ojo, S.O., Bailey, D.P., Brierley, M.L. et al. Breaking barriers: using the behavior change wheel to develop a tailored intervention to overcome workplace inhibitors to breaking up sitting time. BMC Public Health 19, 1126 (2019). https://doi.org/10.1186/s12889-0197468-8

31. Kamau N, Njiru H. Water, Sanitation and Hygiene Situation in Kenya's Urban Slums. J Health Care Poor Underserved. 2018;29(1):321336. doi: 10.1353/hpu.2018.0022. PMID: 29503303.

32. Makaudze EM, Gelles GM. The challenges of providing water and sanitation to urban slum settlements in South Africa. InUnderstanding and Managing Urban Water in Transition 2015 (pp. 121-133). Springer, Dordrecht.

33. Buse K, Mays N and Walt G (2005). Making health policy. Maidenhead, UK, Open University Press.

34. Levine P. Prostitution, race and politics: policing venereal disease in the British empire. New York: Routledge. 2003.

35. Shiffman J, Skarbalo M and Subotic J (2002) Reproductive rights and the state in Serbia and Croatia. Social Science and Medicine 54: 625-42

36. Corburn J, Mberu B, Rajasekharan S, Mitaani YM, MaNe Kampala and Stevenson Raz (2020) Addressing the health needs of urban slum dwellers in the context of COVID -19. Urban Health Community of Practice Webinar

37. Buckley RM (2020) Targeting the World's Slums as Fat Tails in the Distribution of COVID-19 Cases. J Urban Health. 2020;97(3):358364. doi:10.1007/s11524-020-00450-w

38. Miller J, Graham L, Pennington J. The nexus of knowledge and behavior for school-aged children: implementation of Health Education Programs and a Nutritional Symbol System. Asia-Pacific Journal of Health, Sport and Physical Education. 2013 Nov 1;4(3):217-34.

39. Yeretzian JS, Afifi RA. 'It won't happen to me': the knowledge-attitude nexus in adolescent smoking. J Public Health (Oxf). 2009 Sep;31(3):354-9. doi: 10.1093/pubmed/fdp031. Epub 2009 Apr 15. PMID: 19369226.

40. Kredo T, Cooper S, Abrams A, Muller J, Volmink J, Atkins S. Using the behavior change wheel to identify barriers to and potential solutions for primary care clinical guideline use in four provinces in South Africa. BMC health services research. 2018 Dec;18(1):1-2.

41. Zimmerman E, Caetano V, Banay R, Smith J. Evidence review and analysis of provider behavior change opportunities. Breakthrough RESEARCH Final Report. Washington DC: Population Council. 2020

42. Lindbolm CE and Woodhouse EJ (1993) The policy-making process. $3^{\text {rd }}$ Edition. Engelwood Cliffs NJ: Prentice-Hall

43. Lindbolm CE (1959) The science of muddling through. Public Administrative Review 19:79-88

44. Walt G (1994) Health policy: an introduction to process and power. London: Zed Books

Page 14/17 
45. Lipsky M (1980) Street level bureaucracy: dilemmas of the individual in public services. New York: Russell Sage Foundation

46. Figueiras MJ, Ghorayeb J, Coutinho MVC, Marôco J and Thomas J (2021) Levels of Trust in Information Sources as a Predictor of Protective Health Behaviors During COVID-19 Pandemic: A UAE Cross-Sectional Study. Front. Psychol. 12:633550. doi: $10.3389 /$ fpsyg.2021.633550

47. Hussein AS (2012) Message Framing, Source Credibility and Behavioural Intention: An Empirical Investigation of Exclusive Breastfeeding Intention in Indonesia. PhD Thesis, University of Lincoln

48. Ibáñez-Alfonso JA, Hernández-Cabrera JA, Duñabeitia JA, Estévez A, Macizo P, Bajo MT, Fuentes LJ, Saldaña D. Socioeconomic Status, Culture, and Reading Comprehension in Immigrant Students. Frontiers in psychology. 2021 Nov 19;12:752273.

49. Dolean D, Melby-Lervåg M, Tincas I, Damsa C, Lervåg A. Achievement gap: socioeconomic status affects reading development beyond language and cognition in children facing poverty. Learning and Instruction. 2019 Oct 1; 63:101218.

50. O'Mara-Eves A, Brunton G, Oliver S, Kavanagh J, Jamal F, Thomas J. The effectiveness of community engagement in public health interventions for disadvantaged groups: a meta-analysis. BMC public health. 2015 Dec;15(1):1-23.

51. Gilmore B, Ndejjo R, Tchetchia A, De Claro V, Mago E, Lopes C, Bhattacharyya S. Community engagement for CoVID-19 prevention and control: a rapid evidence synthesis. BMJ global health. 2020 Oct 1;5(10): e003188.

\section{Figures}

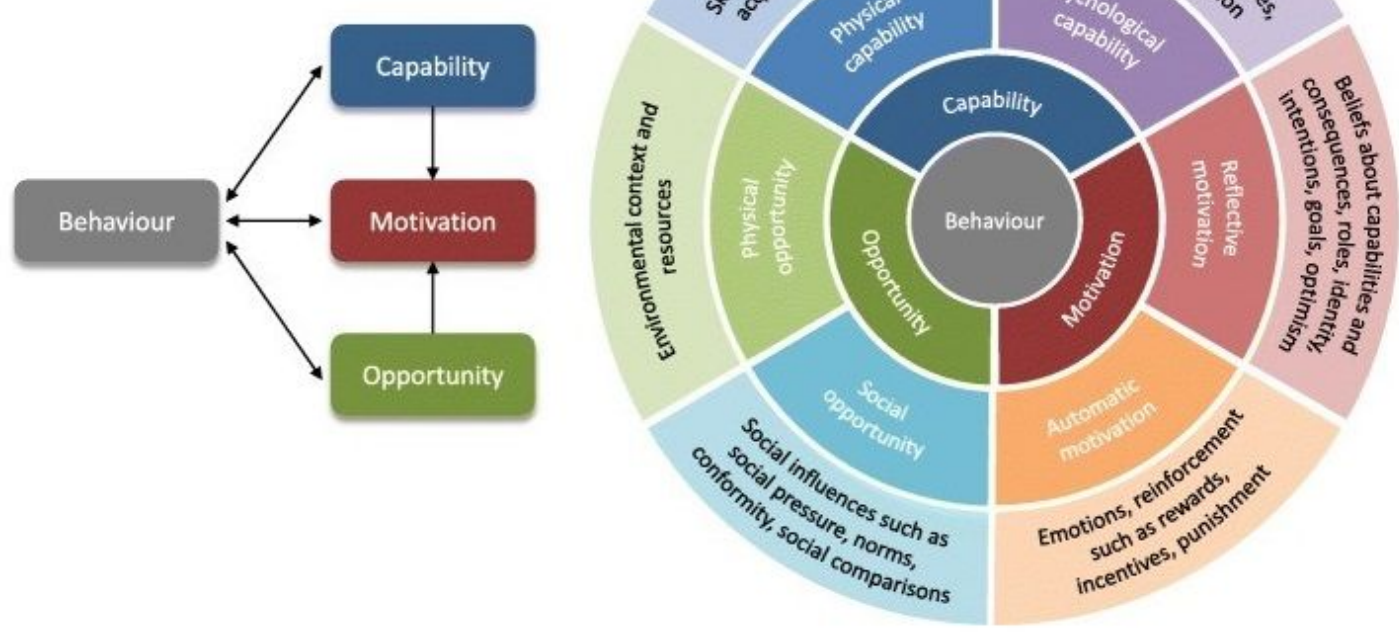

\section{Figure 1}

The COM-B model (McDonagh et. al, 2018) 


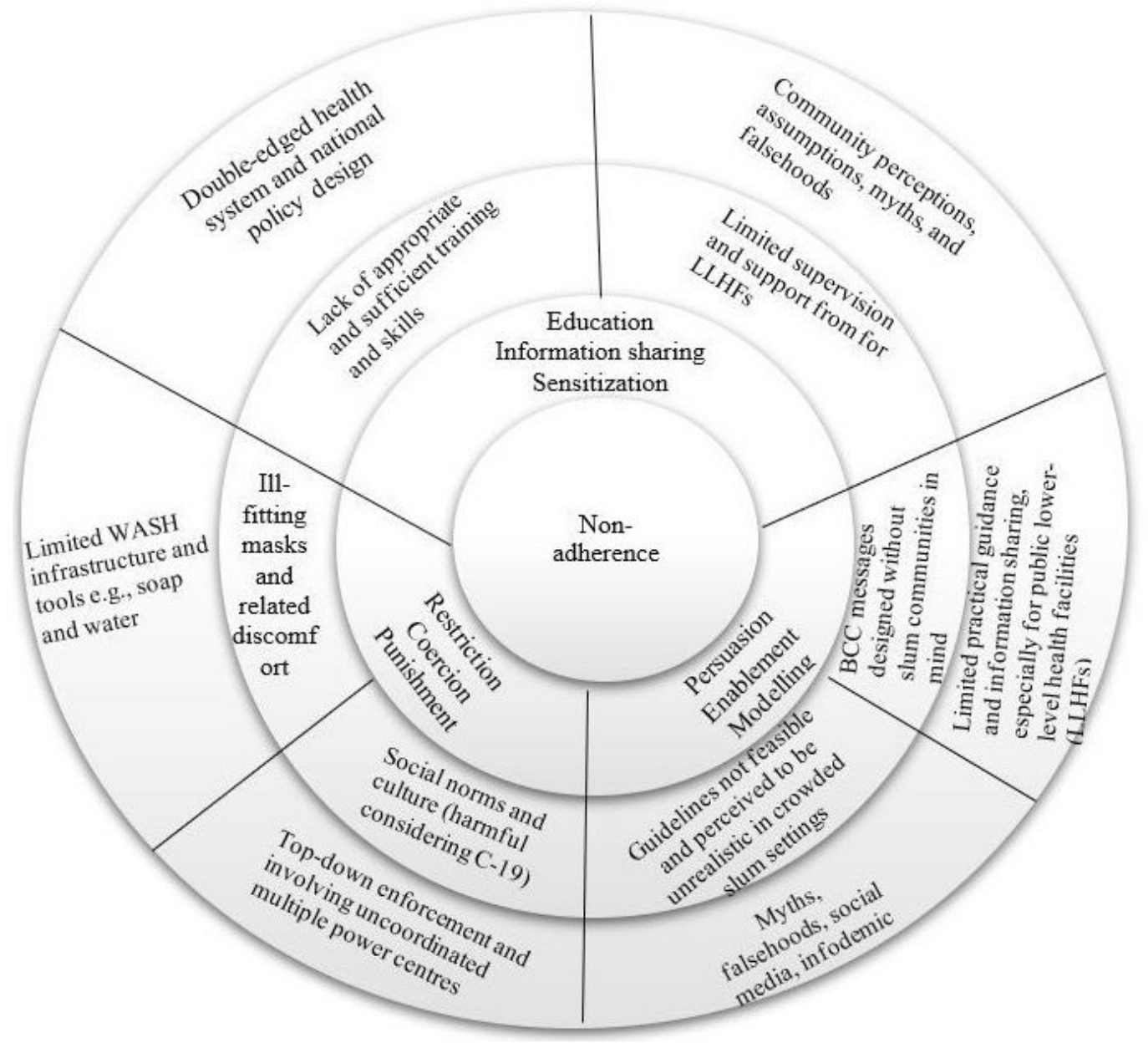

Figure 2

Behavioral Change Wheel informed by the ALERTs study findings - Barriers to adherence 


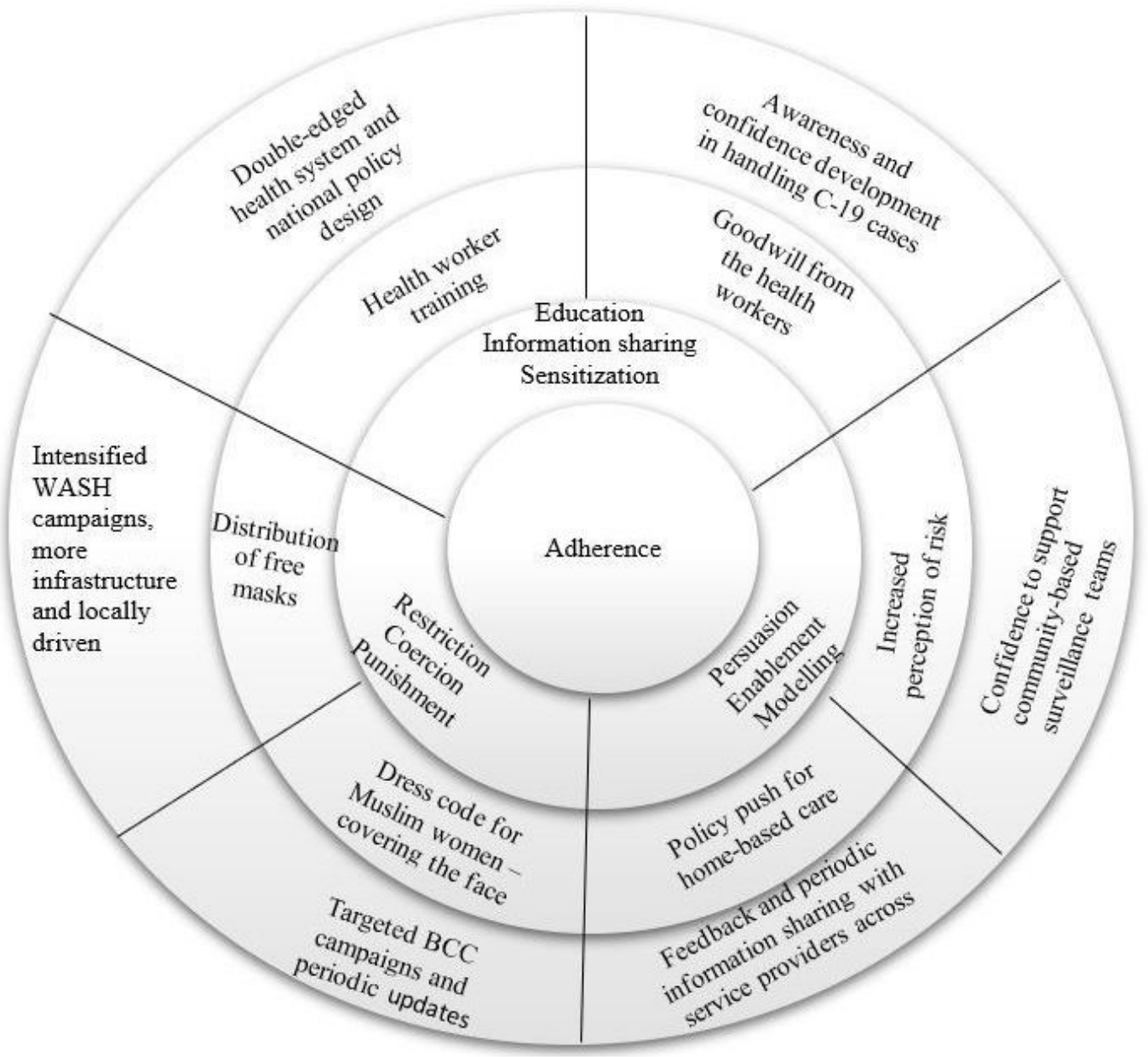

Figure 3

Behavioral Change Wheel informed by the ALERTs study findings - Facilitators of adherence 